\title{
INVESTIGACIÓN/RESEARCH
}

Recibido: 23/09/2012--Revisado: 20/11/2012 Aceptado: 30/01/2013---Publicado: 15/06/2013

\section{EL "PLAN DIRECTOR PARA LA CONVIVENCIA Y MEJORA DE LA SEGURIDAD ESCOLAR" Y SUS ESTRATEGIAS DE DESARROLLO EN LAS REDES SOCIALES}

Ma Verónica de Haro de San Mateo'. Universidad de Murcia. España mvdeharo@um.es

Francisco Lorenzo Solá2. Universidad de Alicante. España. francisco.lorenzo@ua.es

\section{RESUMEN}

La preocupación por las cuestiones relacionadas con la seguridad de los menores ha impulsado la puesta en marcha de diversas iniciativas a cargo del Gobierno de España en colaboración con las Fuerzas y Cuerpos de Seguridad del Estado. Así, con el acuerdo de los sindicatos de enseñanza, las organizaciones de los centros educativos y las asociaciones de padres y madres de alumnos, el Gobierno español aprobó en marzo de 2006 el "Plan para la Mejora de la Convivencia Escolar" con la finalidad de fomentar hábitos y conductas que promovieran la concordia en estos entornos. En el marco de la ejecución de este Plan Director no han pasado inadvertidas las posibilidades de Internet y las Redes Sociales. Describir las acciones emprendidas por los Ministerios del Interior, de Educación y Ciencia e Igualdad del Gobierno de España en colaboración con las Fuerzas y Cuerpos de Seguridad del Estado en materia de seguridad escolar a través de las Redes Sociales es, precisamente, el objetivo de este trabajo.

\footnotetext{
${ }^{1} \mathbf{M}^{\mathrm{a}}$ Verónica de Haro de San Mateo. Licenciada y Doctora en Periodismo por la Universidad Complutense de Madrid. Actualmente es Profesora del Área de Periodismo en la Facultad de Comunicación y Documentación de la Universidad de Murcia y sus principales líneas de investigación son el Desarrollo de la Comunicación Social y la Historia del Periodismo.

mvdeharo@um.es
}

${ }^{2}$ Francisco Lorenzo Solá. Licenciado en Publicidad y Relaciones Públicas por la Universidad de Alicante y Licenciado en Periodismo por la Universidad de Murcia. Actualmente es Profesor Asociado en la Universidad de Alicante y sus principales líneas de investigación son el estudio de la Gestión de la Comunicación en los gabinetes de comunicación como herramienta de las Relaciones Públicas. francisco.lorenzo@ua.es 
PALABRAS CLAVE Redes Sociales; Seguridad escolar; Bullying escolar; Tuenti; Plan Director; Gobierno de España

\title{
THE "DIRECTOR PLAN FOR THE SCHOOL SAFETY" AND ITS DEVELOPMENT'S STRATEGIES IN THE SOCIAL NETWORKS
}

\begin{abstract}
The Government of Spain and the Forces and Bodies of Security of the State have undertaken diverse initiatives related to the safety and the minors. The Spanish government approved in March 2006, the "Director Plan for the eschool safety" (with the agreement of the teaching unions, organizations of schools and associations of parents of students) in order to encourage behaviors that promote harmony in these environments. The aim of our work is to describe the actions undertaken by the Departments of the Interior, of Education and Science and Equality of the Government of Spain (in collaboration with the Forces and Bodies of Security of the State) to promote the school safety across the Social Networks.
\end{abstract}

KEY WORDS Social Networks; School safety; Tuenti; School bullying; Government of Spain; Education; 'Plan Director'

\section{INTRODUCCIÓN}

La Ley Orgánica 2/2006 de Educación establecía - entre los principios que deben inspirar el sistema educativo - la transmisión y puesta en práctica de valores que favorezcan la libertad personal, la responsabilidad, el ejercicio democrático, la solidaridad, la tolerancia, la igualdad, el respeto y la justicia, así como todos aquellos que ayuden a superar cualquier acción discriminatoria. Parece innegable la relación existente entre la educación que reciben los jóvenes y su comportamiento futuro como ciudadanos, constituyendo la escuela una institución básica - junto con la familia - para la integración social, la adquisición de valores y habilidades sociales, así como para la prevención de conductas contrarias a la convivencia. La propia escuela, entorno ideal para el aprendizaje de la convivencia, debiera ser ejemplo de los valores ciudadanos y de seguridad que pretende impulsar, pero esta circunstancia exige un firme compromiso de los poderes públicos y del resto de agentes sociales que deben esforzarse por erradicar cualquier conducta violenta, muy especialmente las derivadas de la violencia entre iguales o el acoso escolar, porque si es importante que los estudiantes desarrollen el nivel de conocimientos adecuado que les permita alcanzar las metas profesionales personal y socialmente deseadas, no lo es menos que los centros educativos proporcionen el ambiente de paz y seguridad en el que los menores puedan formarse y socializarse 
adecuadamente.

Si bien esa labor educativa se concreta a través de los currículos escolares correspondientes y se ha reforzado fundamentalmente a través del impulso a los mecanismos de la tutoría y la mediación, se hace necesario articular fórmulas de coordinación entre la comunidad educativa y los expertos en esta materia para facilitar el intercambio de conocimiento y abordar la solución de los casos más preocupantes. Esta tarea bien podría complementarse con la puesta en práctica de otras actividades que permitieran mejorar el conocimiento que los jóvenes tienen sobre los principales problemas de seguridad ciudadana que les afectan como colectivo y sobre los recursos con los que cuenta la sociedad para la prevención de la delincuencia y la protección de las víctimas.

La comprensión en el ámbito escolar de las funciones de los Cuerpos Policiales debiera contribuir a mejorar la confianza de los jóvenes en los mismos, favoreciendo su implicación en valores vinculados a la seguridad y la colaboración ciudadana así como la denuncia de aquellos actos violentos de los que sean testigos o víctimas. En este contexto, la preocupación por las cuestiones relacionadas con la seguridad de los menores ha impulsado la puesta en marcha de diversas iniciativas en este ámbito a cargo del Gobierno de España en colaboración con las Fuerzas y Cuerpos de Seguridad del Estado. Así, con el acuerdo de los sindicatos de enseñanza, las organizaciones de los centros educativos y las asociaciones de padres y madres de alumnos, el Gobierno español aprobó en marzo de 2006 el "Plan para la Mejora de la Convivencia Escolar" con la finalidad de fomentar hábitos y conductas que promovieran la concordia en estos entornos. Para su puesta en marcha, el Ministerio del Interior se comprometía a cooperar a través de su participación en el Observatorio Estatal de la Convivencia Escolar y la Prevención de Conflictos y reforzando el "Portal para la Convivencia" puesto en marcha por el Ministerio de Educación y Ciencia en Internet. Además, el Consejo Nacional de Seguridad Ciudadana reunido en Madrid en abril de 2006, acordó la constitución de un grupo de trabajo sobre "Juventud y Seguridad" con el objetivo de proponer medidas en el campo de la cooperación policial con las Administraciones Públicas y los agentes sociales para contribuir a abordar las materias de seguridad ciudadana relacionadas con los jóvenes.

\subsection{El Plan Director para la Convivencia y Mejora de la Seguridad Escolar}

En febrero de 2007 se firmaba un acuerdo marco entre el Ministerio de Educación y Ciencia y el Ministerio del Interior denominado "Plan Director para la Convivencia y Mejora de la Seguridad Escolar" 3 , entre cuyos propósitos se encuentran:

- Responder de manera coordinada y eficaz a las cuestiones relacionadas con la seguridad de los niños y jóvenes en la escuela y su entorno, fortaleciendo la cooperación

\footnotetext{
${ }^{3}$ Plan Director para la Convivencia y Mejora de la Seguridad Escolar, Ministerio del Interior, Febrero 2007, Disponible en: http://www.mir.es/edse/plan_director/ Consultado el 5 de febrero de 2011.
} 
policial con las autoridades educativas en sus actuaciones para mejorar la convivencia y la seguridad en el ámbito escolar.

- Impulsar el desarrollo de actividades, charlas y conferencias en los centros escolares dirigidas a los alumnos e impartidas por expertos policiales.

- Mejorar la vigilancia policial en las inmediaciones de los centros escolares.

- Impulsar el establecimiento de mecanismos estables de colaboración entre los expertos policiales y las autoridades docentes, comunidad educativa, asociaciones de padres y madres de alumnos y otras organizaciones.

En cuanto a las líneas de actuación previstas en este Plan Director, se contemplaban como tales:

1. Conferencias y actividades en los centros escolares dirigidas a los alumnos. Se trata de impulsar la realización de charlas, visitas y actividades en centros escolares, dirigidas a los menores y jóvenes e impartidas por miembros de los Cuerpos de seguridad. Las líneas básicas a desarrollar mediante dichas actividades son las siguientes:

a) Facilitar información general a los menores y jóvenes de los principales problemas de seguridad que les afectan como colectivo, prestando especial atención a los siguientes aspectos:

* Consecuencias del acoso escolar en quienes lo padecen, así como de la responsabilidad de todos de combatirlo y denunciarlo.

* Prevención del consumo de drogas y alcohol por los menores y jóvenes.

* Otras cuestiones como bandas juveniles, conductas incívicas y vandálicas, racismo y xenofobia, riesgos de seguridad asociados a las nuevas tecnologías, etc.

* Ventajas de fomentar hábitos y conductas que promuevan la mejor convivencia frente a los conflictos o actos de violencia entre alumnos o con otros miembros de la comunidad educativa.

b) Sensibilizar y orientar a los alumnos sobre aquellos comportamientos que pueden derivar en acciones violentas o delictivas, fomentando los valores de responsabilidad, igualdad, respeto y convivencia.

c) Facilitar a los jóvenes herramientas para la prevención de conflictos y para evitar el riesgo de convertirse en víctimas de determinados delitos (abusos o agresiones sexuales, acoso escolar, acceso a determinados contenidos de Internet, etc.).

d) Inculcar la necesidad de comunicar o solicitar ayuda a los padres y profesores, respecto de los hechos que se produzcan en el interior o fuera del centro escolar, cuando la gravedad de la situación lo aconseje. 
e) Mejorar su conocimiento y confianza en relación con el funcionamiento de las Fuerzas y Cuerpos de Seguridad del Estado y el servicio público que prestan a la comunidad.

2. Mecanismos de comunicación y colaboración con la comunidad educativa. El objetivo fundamental de esta línea de actuación consiste en facilitar a los directivos, al personal docente y las Asociaciones de Padres de Alumnos de los centros que participen en el programa mecanismos de comunicación y colaboración con los expertos policiales en materia de menores y de jóvenes. La finalidad es ofrecerles la posibilidad de concertar reuniones con los expertos para que puedan facilitarles asistencia técnica y apoyo en las siguientes materias:

a) Información general sobre los problemas de seguridad que implican mayor riesgo para los menores y jóvenes, buscando mejorar su conocimiento y la adquisición de pautas de detección de los comportamientos y actitudes que suelen manifestar los jóvenes que los padecen: acoso escolar, consumo y tráfico de estupefacientes, violencia contra la comunidad escolar y los centros (hurtos, robos, vandalismo, etc.), actividad de grupos o bandas violentas de carácter juvenil, abusos y agresiones sexuales, maltrato en el ámbito familiar, riesgos asociados a Internet y las nuevas tecnologías, comportamientos racistas y xenófobos.

b) Mecanismos de prevención que pueden desarrollarse por la comunidad educativa para evitar estos problemas en el ámbito escolar y mejorar la seguridad de los centros.

c) Detección y solución de episodios problemáticos concretos que se planteen en el centro escolar.

d) Asistencia individualizada a padres de menores en situación de riesgo, bien como víctimas o autores de comportamientos violentos en el centro escolar o en los ámbitos en los que se desarrolla su personalidad.

3. Mejorar la vigilancia de las inmediaciones de los centros escolares. Se trata de seguir incrementando los esfuerzos ya iniciados, y que están demostrando una gran eficacia, para la vigilancia del entorno de los centros escolares, en el contexto de la Instrucción 25/2005 de la Secretaría de Estado de Seguridad en materia de respuesta al tráfico minorista y consumo de drogas en los centros educativos y sus entornos. Estos esfuerzos de vigilancia, que se están realizando bajo criterios de colaboración entre las Fuerzas y Cuerpos de Seguridad del Estado y las Policías Locales, deben también encaminarse, en la medida de lo posible, a la detección de las situaciones de consumo y tráfico de drogas y de todo tipo de episodios violentos relacionados con los menores y los jóvenes, buscando mejorar la seguridad global del entorno escolar.

4. Otras actuaciones dirigidas a los alumnos. El objetivo de esta línea de acción es impulsar la realización de otras actividades, de carácter complementario, dirigidas a los menores y jóvenes. Estas actividades se promoverán por la Dirección General de la Policía y de la Guardia Civil, con participación de miembros de los Cuerpos de seguridad, como exhibiciones y demostraciones policiales, jornadas de puertas abiertas 
en las dependencias policiales, convocatorias de concursos (dibujos, redacciones, etc.)

\subsection{Guía metodológica y de contenidos del Plan Director para la Convivencia y Mejora de la Seguridad Escolar}

Las diferentes líneas de actuación que comprende el Plan Director, la sensibilidad de los objetivos perseguidos así como la particularidad de que los principales destinatarios son escolares menores de edad, hacen especialmente delicada la determinación y elaboración de los contenidos a impartir y del material didáctico complementario, siendo especialmente compleja la formación que han de recibir los funcionarios responsables de su desarrollo. Por ello, con la colaboración del Ministerio de Educación y Ciencia, este Plan Director preveía también la elaboración de una "Guía metodológica y de contenidos" así como diverso material pedagógico e institucional de apoyo para su utilización en las conferencias, coloquios y exposiciones, con criterios psicopedagógicos comunes para garantizar la existencia de una única línea en todo el territorio nacional. Además, dicho acuerdo marco preveía la organizarán de Jornadas Técnicas de Formación de Formadores, con la participación de expertos policiales y educativos en las materias relacionadas con la juventud y seguridad y otros agentes sociales, dirigidas a aquellos funcionarios que en las diferentes regiones y zonas se encargaran de la puesta en marcha y posterior coordinación del desarrollo del Plan Director.

El éxito de esta iniciativa durante los cursos escolares de inicial aplicación (2007/2008 y 2008/2009), la gran demanda de las acciones puestas a disposición de la comunidad educativa, así como las recomendaciones favorables del Consejo Nacional de Seguridad Ciudadana, aconsejaron ampliar su periodo de vigencia a los cursos 2009/2010 y 2010/2011 . Además, para complementar las medidas preventivas contempladas en el Acuerdo Marco en Educación para la Mejora de la Seguridad", se ha incorporado al mismo el Ministerio de Igualdad, que asume compromisos en materia de formación y colaboración en las actividades que en relación con la mejora de la convivencia y la seguridad escolar se vienen realizando en el entorno escolar, particularmente las enfocadas a la prevención de la aparición en la escuela de comportamientos proclives a la violencia de género o a cualquier forma de discriminación por razón de sexo u orientación sexual así como en la elaboración y eventual revisión del material educativo a utilizar en las propias actividades objeto del Plan Director y las acciones formativas dirigidas a las Fuerzas y Cuerpos de Seguridad del Estado que se realicen en materia de "Juventud y Seguridad".

\section{METODOLOGÍA}

En el marco de la ejecución de este Plan Director no han pasado inadvertidas las posibilidades de Internet y las Redes Sociales. En este trabajo se pone de relieve la iniciativa denominada "Contigo en Tuenti" puesta en marcha por los Ministerios del Interior, de Educación y Ciencia e Igualdad del Gobierno de España en colaboración con las Fuerzas y Cuerpos de Seguridad del Estado, para la difusión del citado "Plan Director para la Convivencia y Mejora de la Seguridad Escolar". 
Para la descripción de la iniciativa "Contigo en Tuenti" nos hemos servido de la información institucional ofrecida por el gabinete de comunicación de la red social española y la derivada de los documentos fuente elaborados por el Ministerio del Interior y las Fuerzas y Cuerpos de Seguridad del Estado que en la relación de referencias se citan (Plan Director para la Convivencia y Mejora de la Seguridad Escolar. Ministerio del Interior, Febrero 2007; Instrucción 3/2007, de 21 de febrero de la Secretaría de Estado de Seguridad - Ministerio del Interior - Gobierno de España para la puesta en marcha de un "Plan Director para la Convivencia y Mejora de la Seguridad Escolar"; Instrucción número 9/2009, de 21 de septiembre de la Secretaría de Estado de Seguridad - Ministerio del Interior - Gobierno de España para dar continuidad al "Plan Director para la Convivencia y Mejora de la Seguridad Escolar" e Informe anual de contenidos digitales en España 2010)

Lógicamente, hemos analizado pormenorizadamente la página web http://www.tuenti.com/contigo a fin de estudiar el contenido de los mensajes publicados, el lenguaje en el que éstos están expresados así como la tipología temática sobre la que preferentemente dichos consejos centran su atención. A fin de comprender con más detalle los objetivos de la iniciativa y recabar el testimonio de los impulsores del proyecto también nos hemos entrevistado con portavoces de las instituciones involucradas.

\section{ANÁLISIS Y DISCUSIÓN}

El 4 de noviembre de 2010, Alfredo Pérez Rubalcaba, en calidad de Vicepresidente primero del Gobierno y Ministro del Interior, e Ícaro Moyano, responsable de Comunicación y Relaciones Públicas de la popular red social española Tuenti4, presentaban ante los medios la web "Contigo en Tuenti" 5 . Creada para configurar un nuevo espacio de comunicación de las Fuerzas y Cuerpos de Seguridad del Estado al servicio del "Plan Director para la Convivencia y Mejora de la Seguridad Escolar" y con el propósito de acercarse a los jóvenes desde las Redes Sociales para orientarles en cuestiones relativas a la seguridad, la página alcanzaba casi 20.000 amigos en sus primeras veinticuatro horas de vida ${ }^{6}$. La iniciativa, basada en una experiencia previa de la Delegación del Gobierno en Aragón, se inauguraba con cuatro consejos relacionados

\footnotetext{
${ }^{4}$ http://www.tuenti.com

${ }^{5}$ http://www.tuenti.com/contigo

6 “La policía acumula casi 20.000 amigos en Tuenti en poco más de 24 horas", www.abc.es, 6 de noviembre de 2010. Disponible en: http://www.abc.es/20101106/medios-redes/tuenti-policia-201011061245.html Consultado el 6 de noviembre de 2010.
} 
con la identidad real y la protección de la intimidad en la Red7; la seguridad de los perfiles personales y de las claves ${ }^{8}$; los síntomas del maltrato en pareja ${ }^{9}$ y las diferencias entre el tabaco y el cannabis ${ }^{10}$.

\subsection{Las Fuerzas y Cuerpos de Seguridad del Estado Contigo en Tuenti}

Durante sus primeros seis meses de vida, la página www.tuenti.com/contigo ha ofrecido un total de cuarenta y ocho posts ${ }^{11}$ - publicados generalmente los viernes, preludio del fin de semana - relacionados principalmente con el consumo de alcohol y drogas ${ }^{12}$, la seguridad vial ${ }^{13}$, la seguridad en la $\operatorname{Red}^{14}$ o el acoso escolar ${ }^{15}$.

\footnotetext{
7 “¿Cómo sabes que tus amigos de Internet son realmente amigos tuyos? Protege bien tu intimidad y la de los tuyos, ahora y para el futuro", www.mir.es, 3 de noviembre de 2010. Disponible en: http://www.mir.es/DGRIS/Contigo/internet1.html Consultado el 6 de noviembre de 2010.
}

8 "Asegúrate de que no pueden robar tu perfil. Que nadie adivine tu clave y se haga pasar por ti", www.mir.es, 3 de noviembre de 2010. Disponible en: http://www.mir.es/DGRIS/Contigo/internet2.html Consultado el 6 de noviembre de 2010.

9 “¿Cómo noto los primeros síntomas de maltrato en una pareja? Algunos indicios de una relación que hay que cortar", www.mir.es, 3 de noviembre de 2010. Disponible en: http://www.mir.es/DGRIS/Contigo/igualdad1.html Consultado el 6 de noviembre de 2010.

10 “¿El cannabis hace más o menos daño que el tabaco? Mitos y realidades sobre el consumo de drogas”, 3 de noviembre de 2010. Disponible en: http://www.mir.es/DGRIS/Contigo/drogas1.html Consultado el 6 de noviembre de 2010.

${ }^{11}$ Vocablo inglés, traducido al español como "mensaje", generalmente en el contexto de los foros o blogs en Internet.

${ }^{12}$ A modo de ejemplo se pueden citar: “A propósito de fiestas, celebraciones y alcohol...", www.mir.es, 23 de diciembre de 2010. Disponible en: http://www.mir.es/DGRIS/Contigo/trafico1.html Consultado el 23 de diciembre de 2010 y “¿Todavía crees que las pastillas son inofensivas? En torno a las drogas hay muchos mitos que conviene aclarar", www.mir.es, 25 de febrero de 2011. Disponible en: http://www.mir.es/DGRIS/Contigo/drogas3.html Consultado el 25 de febrero de 2011.

${ }^{13}$ A modo de ejemplo se puede citar: “¿Qué me puede pasar si me pillan conduciendo un coche o una moto sin haberme sacado el carné?", www.mir.es, 26 de noviembre de 2010. Disponible en: http://www.mir.es/DGRIS/Contigo/trafico1.html Consultado el 27 de noviembre de 2010.

${ }^{14}$ A modo de ejemplo se puede citar: “Claves para proteger tu intimidad en Internet (y 2)”, www.mir.es, 4 de febrero de 2011. Disponible en: http://www.mir.es/DGRIS/Contigo/proteccion datos2.html Consultado el 5 de febrero de 2011.

${ }^{15}$ A modo de ejemplo se puede citar: "Lo que me pasa, ¿es acoso o es una broma normal entre amigos?", www.mir.es, 19 de noviembre de 2010. Disponible en: http://www.mir.es/DGRIS/Contigo/Acoso1.html Consultado el 20 de noviembre de 2010. 
En ocasiones, con motivo de fechas señaladas, hemos podido constatar cómo se ha focalizado el esfuerzo en una figura delictiva determinada. Así sucede por ejemplo durante las fiestas de Carnaval para recordar el peligro de la suplantación de la identidad en la Red16; a propósito del día de los Santos Inocentes para incidir en el modus operandi de los timos más frecuentes ${ }^{17}$; en el Día Europeo de la protección de Datos para aconsejar sobre cómo preservar la privacidad y la intimidad en Internet ${ }^{18}$ o con motivo del Día de San Valentín, para alertar sobre los síntomas del maltrato. Por su interés, reproducimos el último de los textos citados a continuación:

“Día de San Valentín... ojalá puedas celebrarlo con alguien que te quiere, que te hace sentir bien, que respeta tu libertad. Pero piensa por un momento si la persona con la que estás quiere controlarte, pone mala cara cuando te salen las cosas bien, te llama o te manda mensajes a todas horas, se pone de mal humor cuando quedas con otras personas, intenta separarte de tus amigos o tu familia... No te confundas... eso no es amor. Parece simple, pero no todo el mundo lo tiene tan claro. Cientos de veces hemos oído frases que esconden comportamientos violentos, malos rollos, infelicidad.... Frases que deberían hacernos reaccionar. Por eso hoy os proponemos darles la vuelta: Quien bien te quiere NO te hará llorar. NO sufras por amor, ríete con el amor, disfruta, diviértete. Amores reñidos NO son los más queridos... piensa en ello. Di NO a las relaciones peligrosas" 19

Con el pretexto de la celebración del Día de la Mujer Trabajadora, una nueva publicación recuerda la labor de las agentes de la Policía Nacional y la Guardia Civil que participan en el Plan Contigo en un texto en el que por su tono se contribuye a potenciar también el valor de la igualdad:

"Hoy, como cada 8 de marzo, se celebra internacionalmente el Día de la Mujer Trabajadora y nosotros sólo os queríamos recordar con gran orgullo la imprescindible labor que desarrollan cada día cerca de 7.846 agentes femeninas en la Policía Nacional y 5.089 agentes de la Guardia Civil por la Seguridad en España, en todos los ámbitos que imaginéis, con eficacia, esfuerzo y vocación de servicio al ciudadano. Queremos recordaros que esas casi 13.000 profesionales están dispuestas a ayudaros en sus respectivas especialidades y, por supuesto, también

\footnotetext{
16 "Deja las máscaras para los carnavales", http://www.tuenti.com/contigo, 4 de marzo de 2011. Disponible en: http://www.tuenti.com/contigo Consultado el 6 de marzo de 2011.

${ }^{17}$ A modo de ejemplo se puede citar: “iNo seas pardillo", www.mir.es, 28 de diciembre de 2010. Disponible en: http://www.mir.es/DGRIS/Contigo/timos.html Consultado el 11 de enero de 2011.

18 "Día Europeo de la Protección de Datos. Seis consejos para cuidar tu intimidad", www.mir.es, 28 de enero de 2011. http://www.mir.es/DGRIS/Contigo/proteccion datos1.html Consultado el 29 de enero de 2011.

${ }^{19}$ A modo de ejemplo se puede citar: “Amores reñidos... no, eso no es amor...”, www.mir.es, 12 de febrero de 2011. Disponible en: http://www.mir.es/DGRIS/Contigo/enamorados.html Consultado el 13 de febrero de 2011.
} 
a través del Plan Contigo, en el que intervienen muchas agentes para ayudaros y aportar su experiencia y conocimiento de las diversas materias de seguridad. Así que, además de agradecer su dedicación, queremos que sepáis que, al igual que los otros 110.000 policías y guardias civiles, están donde y cuando les necesitéis para cuestiones de seguridad, dispuest@s a atenderte y ayudarte, con su preparación profesional y especialización necesarias" 20

En su gran mayoría, los usuarios/amigos de la página son - según datos ofrecidos por los responsables de la iniciativa - jóvenes de edades comprendidas entre los 14 y los 19 años que acuden a la web principalmente para confirmar y refrendar sus informaciones/opiniones respecto de las drogas, el acoso escolar o el uso de las Redes Sociales. Aunque muy testimonialmente, existe también otro grupo de usuarios con un perfil totalmente diferente al anterior: el de los padres y los educadores que recurren a esta plataforma con fines pedagógicos con el objetivo de ampliar información sobre los citados temas ${ }^{21}$. El hecho de que la página esté dirigida fundamentalmente al público juvenil justifica el tono coloquial mayoritario de los mensajes publicados. Para ejemplificar esta afirmación reproducimos el mensaje aparecido en marzo de 2011 titulado "El que juega con fuego... se quema":

“Esta semana es la fiesta del fuego, las Fallas valencianas. Y probablemente, tú, como nosotros, habrás oído a tus padres esa frase que te avisa de evitar riesgos innecesarios ¿verdad? Nosotros aprovechamos las Fallas para hablarte de un riesgo absurdo e innecesario: lo que se conoce por sexting, que es cuando compartes fotos 'demasiado sexys' tuyas o de alguien, a través de un mensaje, mail, etc. para sí mism@, para enseñar o bromear con amig@s o como supuesto regalo para su novi@.

Desgraciadamente, cuando las víctimas se hacen esas fotos no se paran a pensar que esa imagen se puede volver en su contra, como un boomerang... Ya sea porque llega a gente que no debe... o porque alguien que tú creías que era $100 \%$ fiable y leal a ti resulta no serlo y reenvía esas imágenes, con el único fin de hacerte daño (iy lo logra!)

Recordad: lo primero, no lo hagas... Tarde o temprano, te puedes acabar arrepintiendo. Cuando compartas una foto, piensa como si lo hicieras con tus padres o tus profesores. Si a ellos no les gusta esa imagen, piensa que es muy probable que a ti, en poco tiempo, tampoco y mucho menos, que la tenga otra gente.

Lo segundo: no lo reenvíes, ni de coña. Y, si recibes foto o video de ese tipo, frena su distribución, hablando con quien te lo ha enviado y apoyando a la víctima (que podrías ser tú, no lo olvides).

\footnotetext{
20 "Día Internacional de la Mujer", http://www.tuenti.com/contigo, 8 de marzo de 2011. Disponible en http://www.tuenti.com/\#m=Page\&func=index\&page key=1 1162 1 Consultado el 8 de marzo de 2011.
}

\footnotetext{
${ }^{21}$ Según los responsables de la iniciativa, el número de consultas atendidas ha alcanzado la cifra de 3.500.
} 
Y lo tercero: si conoces a alguien que quiere compartir imágenes íntimas de otra persona para hacerle daño, dile que se lo piense... Aparte de por ser una persona como se debe ser, porque se estará metiendo en un lío muy serio con nosotros y con la Ley" 22

Con la intención de mejorar la página y lograr un mayor feedback con los usuarios, en enero de 2011 se convoca un concurso de ideas ${ }^{23}$. Las aportaciones deben estar relacionadas con la mejora de la seguridad y la convivencia, pero pueden ser muy variadas: desde un contenido o sección fija para la web a una aplicación para el móvil que sirva para potenciar la seguridad de los jóvenes. El premio para los ganadores consiste en disfrutar una jornada con 10 amigos o familiares en una de las sedes de los grupos de operaciones especiales, ver cómo entrenan y se preparan, y conocer las claves de su trabajo. El 28 de febrero se habían recibido cerca de 200 propuestas y un mes después se alcanzaba un centenar más. El 25 de marzo se anuncian los nombres de los ganadores así como sus propuestas: la posibilidad de que la página ofrezca testimonios directos de policías y guardias civiles en el ejercicio de su labor o la creación de una sección de preguntas y respuestas frecuentes. A pesar de lo acertado de las propuestas, la página no ha incorporado estas sugerencias.

\subsection{El audiovisual como recurso didáctico}

En este punto cabe destacar que la página no sólo muestra mensajes de texto sino también material audiovisual, en su mayor parte elaborado por instituciones como la Dirección General de Tráfico, el Instituto Nacional de Tecnologías de la Comunicación o la Fundación de Ayuda contra la Drogadicción. Entre los vídeos aparecidos destacan el trabajo titulado "Think before you post" 24 que alerta de los riesgos de publicar

\footnotetext{
22 "El que juega con fuego... se quema", www.tuenti.com/contigo, 18 de marzo de 2011 Disponible en: www.tuenti.com/contigo Consultado el 22 de marzo de 2011.

23 "Contigo tenemos mejores ideas. Mándanos tu propuesta y lleva a tus amigos a conocer nuestras unidades de élite", www.mir.es, 6 de enero de 2011. Disponible en: http://www.mir.es/DGRIS/Contigo/propuesta.html Consultado el 11 de enero de 2011.

24 Disponible en: http://www.tuenti.com/\#m=Video\&func=view videos\&collection kev=13-1 1162 1-1446 Consultado el 30 de enero de 2011.La traducción del título al castellano sería: "Piensa antes de publicar". Este vídeo es el más consultado de la página. A la fecha de cierre de este trabajo se contabilizaban un total de 2894 reproducciones. Los esfuerzos institucionales realizados para concienciar a los menores sobre los riesgos del uso indebido de las Redes Sociales han sido significativos. Ya en febrero de 2007, el Ad Council de EEUU junto con el Departamento de Justicia y el National Center for Missing \& Exploited Children, lanzaron la campaña "Think Before You Post", dirigida específicamente a los menores para promover el uso responsable de las Redes Sociales. Con un formato similar al de un spot publicitario, la campaña ha sido traducida y adaptada en muchos países occidentales, incluido el nuestro, alcanzando un gran notoriedad. El vídeo al que nos referimos es precisamente la traducción al castellano de esa iniciativa estadounidense. En España, el Defensor del Menor de la Comunidad de Madrid propició el lanzamiento de un anuncio similar titulado "Piensa antes de colgar tu imagen en Internet". Del mismo modo, e incidiendo especialmente en la protección datos de carácter personal, la Agencia de Protección de Datos de Madrid
} 
información personal en la Red, el que recoge la visita de los ganadores del concurso de ideas citado anteriormente a la unidad $\mathrm{GEO}^{25}$ o uno más reciente que incide en la peligrosidad de conducir bajo los efectos del alcohol.

A la fecha de cierre de este trabajo, la página "Contigo en Tuenti" ha conseguido reunir un total de 60.000 amigos, lo que convierte a las Fuerzas y Cuerpos de Seguridad del Estado en la institución pública española con más seguidores en las Redes Sociales. El éxito de esta iniciativa - al menos en términos de acogida - contrasta con el de la web puesta en marcha en idéntica plataforma por el Ministerio de Justicia en febrero de 2011 titulada "Justo x ti" 26 , que tan solo ha logrado unos pocos cientos de amigos.

\section{CONCLUSIONES}

El estudio titulado "Menores y Redes Sociales", recientemente publicado por los profesores de la Universidad de Navarra Xavier Bringué y Charo Sádaba para el Foro "Generaciones Interactivas" de Fundación Telefónica, revela que más de una tercera parte de los jóvenes internautas españoles entre 10 y 18 años administra un perfil en una red social y que un 35\% tiene más de uno, lo que sitúa a este último grupo como usuarios avanzados de este tipo de herramientas de comunicación 2.0. De la lectura de este estudio se desprende que más del $70 \%$ de los menores internautas españoles son usuarios habituales de redes sociales.

Por este motivo, no es de extrañar que el "Plan Director para la Mejora de la Convivencia y la Seguridad Escolar" alentado por los Ministerios del Interior, de Educación y Ciencia e Igualdad del Gobierno de España en colaboración con las Fuerzas y Cuerpos de Seguridad del Estado para formar y sensibilizar a los jóvenes sobre la seguridad en Internet, el acoso, el cyberbullying, las drogas, la integración social o la violencia de género entre otros temas, se extienda también desde hace unos meses a través de la red social española Tuenti tal y como hemos intentado explicar en este trabajo.

Es evidente que las Redes Sociales han transformado el modo a través del cual las instituciones públicas se acercan a los ciudadanos. Ciertamente, las Administraciones Públicas que trabajan en el ámbito de la seguridad han visto en las Redes Sociales un vehículo ideal para fomentar el desarrollo de sistemas de información que ayuden a difundir sus iniciativas institucionales, formativas y de sensibilización en los ámbitos de la prevención y/o la superación de situaciones de riesgo o inseguridad, en cualquiera de

\footnotetext{
puso en marcha la campaña “¿Por qué hacerlo en Internet?”. Otras administraciones públicas han llevado a cabo acciones similares, como la Agencia Vasca de Protección de Datos que tradujo una exitosa acción del gobierno noruego titulada "¿Anonimato? ¡Tú decides!".

${ }^{25}$ Grupo Especial de Operaciones del Cuerpo Nacional de Policía de España especializado en operaciones de alto riesgo.

${ }^{26}$ http://www.tuenti.com/justoxti
} 
sus manifestaciones para acercarse al público juvenil. La Sociedad de la Información vive permanentemente conectada. Por ello, el Gobierno de España, a través de sus distintas administraciones, ha potenciado el uso de los nuevos medios consciente de los profundos cambios que están motivando las Nuevas Tecnologías de la Información y de la Comunicación.

La puesta en marcha de páginas web para acercar a los ciudadanos las actuaciones gubernamentales fue el primero de los pasos de las distintas administraciones en este sentido. Consolidada la experiencia web, las instituciones públicas de nuestro país no han dudado en habilitar espacios institucionales propios en redes sociales como Facebook y Tuenti o en otras plataformas como You Tube para potenciar su política informativa y acercarse a una sociedad cada vez más familiarizada con las nuevas tecnologías.

\section{REFERENCIAS}

BRINGUÉ SALA, Xavier, SÁDABA, Charo \& RODRÍGUEZ PARDO, Julián (2008). La generación interactiva frente a un nuevo escenario de comunicación. Retos sociales y educativos, Consejo Audiovisual de Navarra, Pamplona.

BRINGUÉ, Xavier \& SÁDABA, Charo (2011). Menores y redes sociales, Fundación Telefónica, Madrid.

CASTELLS, Manuel (2006). La sociedad red: Una visión global, Alianza Editorial, Madrid.

DEL RÍO PÉREZ, Jorge, SÁDABA, Charo \& BRINGUÉ SALA, Xavier (2010). “Menores y redes ¿sociales?: De la amistad al ciberbullying". Revista De Estudios de Juventud, 88 (Juventud y nuevos medios de comunicación), pp. 115-12. Disponible en: http:// www.injuve.es/contenidos.downloadatt.action?id=1880411574 Consultado el 6 de febrero de 2011.

LOSADA DÍAZ, José Carlos (2004). Gestión de la comunicación en las organizaciones, Ariel, Barcelona, 2004.

MARTÍN BARBERO, Jesús (2001). “Transformaciones comunicativas y tecnológicas de lo público", Metapolítica, no 17, pp. 46-55.

MARTÍNEZ SOLANA, Yolanda (2004). La comunicación institucional. Análisis de sus problemas y soluciones, Fragua, Madrid.

MOLINA, José Luis (2001). El análisis de las redes sociales. Una introducción, Edicions Bellaterra, Barcelona.

SÁEZ VACAS, Fernando (2004). La red universal digital, Editorial Ramón Areces, Madrid. SÁNCHEZ BURÓN, Alfonso \& FERNÁNDEZ MARÍN, Ma Poveda (2010). Generación 2.0, 2010. Hábitos de los adolescentes en el uso de las redes sociales, Universidad Camilo José Cela, Madrid.

SOTELO ENRÍQUEZ, Carlos (2001). Introducción a la comunicación institucional, Ariel, Barcelona. 
Instrucción 3/2007, de 21 de febrero de la Secretaría de Estado de Seguridad (Ministerio del Interior. Gobierno de España) para la puesta en marcha de un "Plan Director para la Convivencia y Mejora de la Seguridad Escolar".

Instrucción número 9/2009, de 21 de septiembre de la Secretaría de Estado de Seguridad (Ministerio del Interior. Gobierno de España) para dar continuidad al "Plan Director para la Convivencia y Mejora de la Seguridad Escolar".

Informe anual de contenidos digitales en España 2010, www.red.es Disponible en:http:/ / www.red.es/media/registrados/201011/1290073066269.pdf?aceptacion=d58d bc0adecd9cc3a237adc7c6ab2169 Consultado el 15 de febrero de 2011.

Plan Director para la Convivencia y Mejora de la Seguridad Escolar. Ministerio del Interior, Febrero 2007, Disponible en: http://www.mir.es/edse/plan_director/ Consultado el 5 de febrero de 2011.

Páginas web

http://www.tuenti.com/contigo

http://www.mir.es

http://www.tuenti.com/justoxti

$\mathbf{M}^{\mathrm{a}}$ Verónica de Haro de San Mateo. Licenciada y Doctora en Periodismo por la Universidad Complutense de Madrid. Actualmente es Profesora del Área de Periodismo en la Facultad de Comunicación y Documentación de la Universidad de Murcia y sus principales líneas de investigación son el Desarrollo de la Comunicación Social y la Historia del Periodismo.

mvdeharo@um.es

Francisco Lorenzo Solá. Licenciado en Publicidad y Relaciones Públicas por la Universidad de Alicante y Licenciado en Periodismo por la Universidad de Murcia. Actualmente es Profesor Asociado en la Universidad de Alicante y sus principales líneas de investigación son el estudio de la Gestión de la Comunicación en los gabinetes de comunicación como herramienta de las Relaciones Públicas. francisco.lorenzo@ua.es 